\title{
Concordance between Symptoms Reported By Patients and Signs Elicited By Orthopedic Surgeons
}

\author{
Afzal Hussain ${ }^{1}$, Saleem Bashir ${ }^{2}$ and N Rehan ${ }^{3 *}$ \\ ${ }^{1}$ Chief Consultant and Orthopaedic Surgeon, KKT Spine Centre, Pakistan \\ ${ }^{2}$ Consultant Orthopedic Surgeon, KKT Spine Centre, Pakistan \\ ${ }^{3}$ Research Consultant, KKT Spine Centre, Lahore, Pakistan
}

Received: December 20, 2017; Published: January 09, 2018

*Corresponding author: Dr. N. Rehan, Chief Executive, Research Associates, 275/C, Askari - X, Lahore cantt, Pakistan, Tel: 0300843 9877;

Email:nrehan@yahoo.com

\section{Abstract}

Objectives: To explore concordance between symptoms and signs among orthopaedic patients.

Methodology: 100 consecutive patients complaining of pain in joints of neck, upper/middle back, lower back, shoulders, elbow, wrist, hands, hips, knees, ankles and feet were included in the study. History taken by Medical Officer and a Psychologist was followed by physical examination by an Orthopedic Surgeon.

Results: The mean age of 60 males and 40 females was $50.5 \pm 5.1$ years; being $50.4 \pm 17.0$ years among males and $50.6 \pm 11.8$ years in females $(P=0.94)$. The overall concordance between the pain spots reported by patients and tender points elicited by attending surgeon was $31.3 \%$. The patients with low back pain contributed $59.2 \%$ of the total concordance. The combined concordance observed in neck, ribs, arms, wrists, hands, hips, legs and feet was $16.6 \%$.

Conclusion: In view of 31.3\% concordance between the Symptoms and Signs, more importance should be given to findings of physical examination than the symptoms.

Abbreviations: BSI: Brief Symptom Inventory; GAF: Global Assessment of Functioning; LPR: Laryngo Pharyngeal Reflux

\section{Introduction}

Symptoms are a departure from normal function or feeling which is noticed by patient. These are subjective phenomena observed by the patient and cannot be measured directly. On the other hand, a sign is objectively observable by others. Any objective evidence of a disease, such as blood in the stool, a skin rash, is a sign as it can be recognized by the doctor, nurse, family members apart from the patient. However, lower-back pain, stomachache or fatigue, is only detected or sensed by the patient and others only know about only when the patient tells them [1]. Historically, patients and physicians used to participate more equally in identifying signs and symptoms during a medical consultation. Over the last 200 years as medicine advanced and diagnostic techniques developed, the identification of signs became more and more the doctor's domain [2].The importance of "Signs" as a part of physical examination started with the discovery of "percussion" by Auenbruggerin 1760 and its dissemination by Corvisart in 1808 and development of auscultation in 1816 by Laennec [3].

During the 20th century, hundreds of new devices and techniques were created to identify signs. It was during this period in modern medical history that the term 'symptoms" became known as something the patient notices [3]. There are conflicting reports regarding concurrence between symptoms and signs of various diseases. Lyle et al. [4] studied 254 patients of low back pain to study the relationship of physical examination finding and self-reported symptoms severity in patients with degenerative lumbar conditions. They observed that association between the impairment-based movement pattern and self- reported severity are consistent with the patho-anatomy of degenerative spinal conditions. On the other hand, Piersma and Boes [5] carried out a study on two rating scales on 200 adult psychiatric inpatients at admission and discharge from, the hospital. Patients rated their own psychological symptoms on the Brief Symptom Inventory (BSI), and clinicians rated patients' psychological, social, and occupational functioning on the Global Assessment of Functioning (GAF) Scale.

Analyses indicated no significant relationships between symptom distress reported by patients and global functioning rated by clinicians. According to Barsky [6] patients' recollections of their past symptoms, illnesses, and episodes of care are often inconsistent 
from one inquiry to the next. Patients frequently fail to recall (and therefore underreport) the incidence of previous symptoms and events; tend to combine separate, similar occurrences into a single, generic memory; and falsely recall medical events and symptoms that did not in fact occur. This unreliability of recall is affected by personality characteristics and by the patient's current state at the time of recall. Thus, current anxiety or depression and pain or bodily distress fosters the recall of symptoms and events that are not recalled when the patient is more comfortable. Joint pains are a common problem presenting to primary care clinicians as well as orthopaedic surgeons.

The typical scenario in orthopaedic clinics is that as soon as a patient reports with a complaint of any joint pain, all attention is focused only on physical and radiological investigations of only that joint, subjecting the patient to a dose of radiation and ignoring attention to other parts of locomotive system. A rational approach demands a more selective use of investigations [7]. A rational approach demands a more selective use of investigations [7]. A review of the literature reveals that studies on concordance of symptoms reported by patients and signs elicited on physical examination are non-existent in Pakistan. The present study was, therefore, undertaken to assess the existence of such a concordance.

\section{Material and Methods}

Table 1: Complaints of the patients.

\begin{tabular}{|c|c|c|c|}
\hline S. No. & Complaint & No. of Cases & Percent \\
\hline 1 & Neck Pain & 17 & 17 \\
\hline 2 & Low Back Pain & 53 & 53 \\
\hline 3 & Shoulder Pain & 5 & 5 \\
\hline 4 & Hip Pain & 5 & 5 \\
\hline 5 & Knee Pain & 12 & 12 \\
\hline 6 & Pain Legs & 4 & 4 \\
\hline 7 & Pain Arms & 1 & 1 \\
\hline 8 & Foot Drop & 2 & 2 \\
\hline 9 & Cerebral Palsy & 1 & 1 \\
\hline \multicolumn{3}{|c|}{ Total } & 100 \\
\hline
\end{tabular}

The medical records of 100 consecutive patients, who reported to us for treatment of joint pains during 2014, were retrieved.
The presenting symptoms of these patients are shown in Table 1. According to the protocol of our Centre, attention is not restricted to that particular joint but information is sought in respect of pain at joints of lower/middle back (thoraco-lumbar region), lower back (Sacral region), neck, ribs, shoulders, arms, elbow, wrist, hands, hips, legs, knees, ankles and feet. During history taking the pain spots reported by patients are recorded on this diagram by Medical Officers/Psychologists. Then during examination, the attending surgeon plots the tender points elicited by him/her on a similar bur separate diagram. The attending surgeon elicits the presence of tender points through palpation. For cervical spine, transverse processes are palpated on both sides from $\mathrm{C} 2$ to $\mathrm{C} 6$ for each vertebra and spinous process is palpated for $\mathrm{C} 7$ vertebra.

For thoracic and lumbar spine, spinous processes are palpated for each vertebra. For sacral spine posterior surface is palpated. SI joint is palpated on posterior joint line, ankle joint is palpated on deltoid ligament and lateral collateral ligament, knee joint is palpated on site of attachment of medial collateral ligament, hip joint is palpated on greater trochanter, sternoclavicular and shoulder joints are palpated on anterior joint lines, elbow joint is palpated on lateral and medial side of olecranon, wrist joint is palpated on joint line dorsally and on radial and ulnar styloid processes and small joints of hands and feet are palpated on respective joint lines. For the present study "Concordance" was defined as the number of pain spots reported by the patient on a particular site and verified by attending orthopaedic surgeon on the same patient on the same site.

\section{Results}

There were 60 males (60\%) and 40 females (40\%).Their age ranged from 13 to 79 years The overall mean age was $50.5 \pm 5.1$ years being $50.4 \pm 17.0$ years among males and $50.6 \pm 11.8$ years in females. There was no statistically significant difference between the mean age of male and females $(\mathrm{P}=0.94)$. The presenting complaints of study population are shown in Table 1 . The protocol at our centre calls for a complete information and examination of all major joints of a patient (Table 2). During history taking, each patient was asked about the presence of pain in joints mentioned in Table 2. .During examination, attending surgeon checked all these joints to elicit the presence of tender points.

Table 2: Concordance between Patient's Statement and Surgeon's Examination.

\begin{tabular}{|c|c|c|c|c|c|}
\hline \multirow{2}{*}{ No. } & \multirow{2}{*}{ Site of Pain } & \multicolumn{2}{|c|}{ No. of Sites } & \multicolumn{2}{|c|}{ Concordance } \\
\hline & & Patient's Statement & Surgeon's Examination & No. & $\%$ \\
\hline 1 & Upper Middle Back (Thoraco-lumber region) & 14 & 40 & 10 & 71.4 \\
\hline 2 & Lower Back (Sacral Region) & 67 & 71 & 54 & 80.3 \\
\hline \multicolumn{2}{|r|}{ Concordance between No's; $1-2$} & 81 & 111 & 64 & 79.1 \\
\hline 3 & Neck & 35 & 38 & 20 & 57.1 \\
\hline 4 & Ribs & 3 & 0 & 0 & 0 \\
\hline 5 & Shoulders & 32 & 18 & 2 & 6.2 \\
\hline 6 & Arms & 8 & 1 & 0 & 0 \\
\hline 7 & Elbow & 10 & 4 & 0 & 0 \\
\hline 8 & Wrist & 6 & 1 & 0 & 0 \\
\hline
\end{tabular}




\begin{tabular}{|c|c|c|c|c|}
\hline 9 & Hands & 2 & 0 & 0 \\
\hline 10 & Hips & 37 & 2 & 2 \\
\hline 11 & Legs & 41 & 9 & 3.4 \\
\hline 12 & Knees & 44 & 22 & 15 \\
\hline 13 & Ankles & 26 & 3 & 24.1 \\
\hline 14 & Feet & 20 & 0 & 0 \\
\hline & Concordance (No.3-14) & 264 & 98 & 0 \\
\hline & Overall Concordance (No. 1-14) & 345 & 209 & 16.8 \\
\hline
\end{tabular}

*sThe figures under Patient's Statement and Surgeon' Examination are percentages out of study subjects.

* Concordance" was defined as the number of pain spots reported by the patient on a particular site and tender areas elicited by attending orthopedic surgeon on the same patient on the same site.

The patients reported 345 sites of pain while the attending orthopaedic surgeon elicited 209 tender points. The overall concordance between the pain spots reported by patients and tender points elicited by attending surgeon was $31.3 \%$. The maximum concordance was noted at Sacral region (80.3\%) followed by 71.4 $\%$ in Thoraco-lumbar region. The minimum concordance was seen in the region of hip joint (5.4\%).There was no concordance at the sites of ribs, arms, elbows, wrists, hands, and feet. A strong concordance was observed only among cases of low back pain. If these cases are eliminated from analysis the concordance level falls to a lower level of $16.6 \%$.

\section{Discussion}

The results of the present study indicate very little concordance $(32.3 \%)$ between patient's reported sites of pain and surgeon's elicited sites. Similar situation has been observed in studies from other countries [7-10].

Sullivan et al. [7] carried out a retrospective analysis of data collected from359 subjects recruited from different sites based on either a history of dry eye or no history of dry eye over a 2-year period prior to presentation in an approximate ratio of 2:1 dry eye versus normal. No consistent relationship was found between common signs and symptoms of dry eye disease. Each type of measurement provides distinct information about the condition of the ocular surface. They have argued for a consensus of clinical signs that better reflect all aspects of the disease. Laryngopharyngeal reflux (LPR) is diagnosed by the presence of laryngeal signs and symptoms.

Some studies have noted that signs and symptoms may be non-specific and may have poor correlation. However, many such studies were either observational or had short-term followup. Therefore, Qadeer et al. [9] from Mayo Clinic, USA conducted subgroup analysis of a prospective concurrent controlled study with one year follow-up to ascertain the correlation between signs and symptoms. They found very poor correlation between signs and symptoms of LPR, particularly when monitoring therapeutic

outcomes. Moreover, whatever element of the patient's history, health care provider intends to use for diagnostic purposes, misunderstandings, forgetfulness, cognitive problems such as telescoping and re-composition, and social acceptability biases all need to be taken into account [10]. The results of the study suggest that in view of small concordance between the Symptoms and Signs in orthopaedic patients, more importance should be given to findings of physical examination.

\section{References}

1. Liddell HG, Scott R (1999) Sumptoma : A Greek-English Lexicon.

2. Nordqvist C (2014) What are symptoms? What are signs? Medical News.

3. Devroede G (1992) Constipation-a sign of a disease to be treated surgically, or a symptom to be deciphered as nonverbal communication? J Clin Gastroenterol 15(13): 189-191.

4. Lyle MA, Manes S, McGuinness M, Ziaei S, Iversen MD (2005) Relationship of physical examination finding and self-reported symptoms severity and physical function in patients with degenerative lumbar conditions. Physical Therapy 85(2): 120-133.

5. Piersma HL, Boes JL (1995) Agreement between patient self-report and clinician rating: Concurrence between the bsi and the gap among psychiatric inpatients. Journal of Clinical Psychology 51: 153-157.

6. Barsky AJ (2002) Forgetting, fabricating and telescoping: the instability of the medical history. Arch Intern Med 162(9): 981-984.

7. Sullivan BD, Crews LA, Messmer EM, Foulks GN, Nichols KK, et al. (2014) Correlations between commonly used objective signs and symptoms for the diagnosis of dry eye disease: clinical implications. ActaOphthalmol 92(2): 161-166.

8. Viso E, Rodriguez-Ares MT, Abelenda D, Oubina B, Gude F (2012) Prevalence of asymptomatic and symptomatic meibomian gland dysfunction in the general population of Spain. Invest Ophthalmol Vis Sci 53(6): 2601-2606.

9. Qadeer MA, Swoger J, Milstein C, Hicks DM, Ponsky J, et al. (2005) Correlation between symptoms and laryngeal signs in laryngopharyngeal reflux. Laryngoscope 115: 1947-1952.

10. Cosgrove J B R (1955) An evaluation of the importance of symptoms, signs and spinal fluid findings in the diagnosis of poliomyelitis in the absence of paralysis. CMAJ 72(11): 808-811. 


$\begin{array}{ll}\text { AIOMEDICAL } & \text { Assets of Publishing with us } \\ \text { RESEARCHES } & \text { Global archiving of articles }\end{array}$

